\title{
Feature Selection Criterion for Gravity Matching Navigation
}

\author{
Abubakar Usman Sambo ${ }^{1}$, Li Shi Xin ${ }^{2}$ \\ Tianjin University of Technology and Education, Department of Electronics Engineering. Tianjin China
}

\begin{abstract}
Gravity navigation is a new type of physical field passive navigation, its remarkable characteristic is its precision and reliability are closely related to the selected digital map area. The digital gravity map usually includes the position and the field value information, and is stored in the discrete point form. How to describe the characteristic of the digital map quantitatively and comprehensively is not uniform. In this paper, from the mathematical statistics and the random field theory of the standard deviation, roughness, entropy and relative distance concept is applied to digital gravity map, as a measure of gravity map feature parameters of the calculation methods on the digital map. After the simulation of several matching algorithms in different gravity field, the experience criteria of the map feature region selection is summed up. In order to satisfy the selection criteria, the matching probability is greater than 95\%, and the mean value of the matching error is controlled within 0.3 cell grids. It can be seen that the selection criteria of the feature region can meet the requirements of general engineering applications, and can be used as reference for the actual gravity navigation
\end{abstract}

Keywords: Gravity navigation; Characteristic region; Gravity entropy; Correlation distance.

\section{Introduction}

Gravity navigation, as a new type of geophysical field, has received extensive attention in recent years. It can be combined with the inertial navigation system, which can adjust the inertial navigation system on a regular basis, and adjust the accumulation error, so as to realize the long time high precision navigation. Gravity navigation technology in the acquisition of gravity information without energy radiation, with good concealment. The inertial/gravity matching integrated navigation system gets the best matching position by a certain matching algorithm, and then the inertial navigation system is corrected [1]. The result of the gravity navigation solution is closely related to the gravity field of the gravity map, and it is not suitable for navigation in some regions [7]. In order to ensure the accuracy of the gravity matching, the selection criteria of the matched feature region is needed.

\section{Basic Strategies for Gravity Navigation}

The basic idea of the gravity / inertial navigation is by way of mutual compensation of gravity and inertia, namely first by INS output drift valuation information and systems to provide a reference region gravity, then the location of the output in the region of gravity matches correction INS [ 3 ] [ 4 ]. Due to gravity map database of the confined space and gravity map features, gravity matching is often used for intermittent matching, i.e., matching only in a specific area, gravity navigation system matching process diagram is as shown in Figure 1.

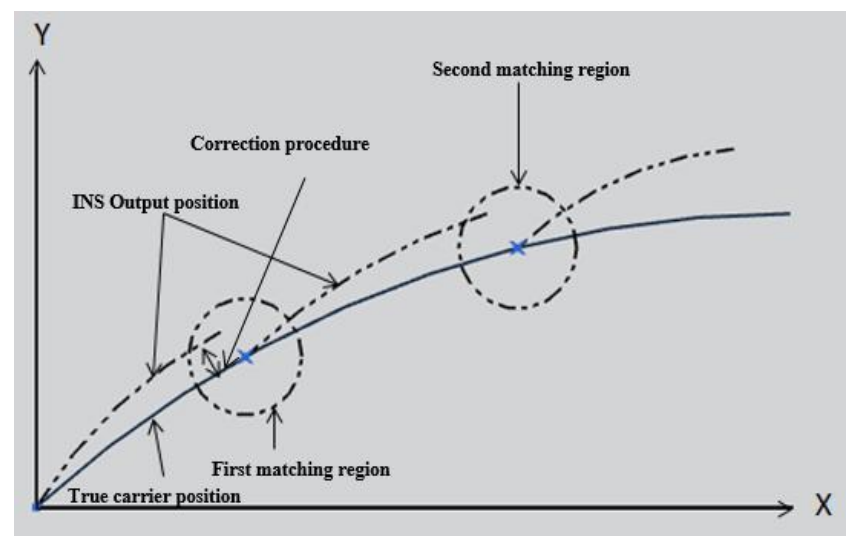

Figure 1: Structure and process of the gravity Navigation System

In the matching process, according to the characteristics of gravity field in different regions, in order to accurately obtain real time high precision and high probability of matching positioning result, the different matching parameters and matching strategies should be adopted [9 ]. Here, the main pre-selected two regions are as follows, one is the characteristic region, and the other is the matching region. Regional characteristics including all used to guide the location where the output and the true position, only the feature regions in the right location is feasible, regional characteristics of sampling frequency, matching step and matching probability plays a decisive role. Matching area is an area which is limited to the relevant range when the matching algorithm is performed. It can reduce the amount of matching operations and improve the matching accuracy. The matching region is an area which is centered on the position of the inertial navigation system.

It changed with the specific output of the inertial navigation system. The range is mainly determined by the inertial navigation position drift error estimation. In general, the feature area should include the matching region. 


\section{International Journal of Science and Research (IJSR) \\ ISSN (Online): 2319-7064}

Index Copernicus Value (2013): 6.14 | Impact Factor (2014): 5.611

Gravity feature selection is an important aspect of gravity matching technology. In order to make the gravity matching system produce effective performance, it is necessary to select the appropriate matching features in advance. Feature selection, which is directly related to the effectiveness of the matching results, can make the gravity navigation system to obtain a higher positioning accuracy and capture the strange rate [7]. General steps for feature selection area are as follows, to determine the size of the regional characteristics;

2. Calculate the characteristic parameters of a certain characteristics of the region; 3 . Combining with the field sensor performance and carrier motion, judge the region matching feasibility.

Regional characteristics cannot be selected too large, In general the calculated characteristic parameters cannot reflect the actual carrier operating region of the characteristics of gravity field; regional characteristics cannot be too small, regional characteristics should guarantee the matching length of INS indicated trajectory and real trajectories are within the range. However, the matching length is usually related to the parameters of the feature region, and the characteristic parameters are related to the region size, so the size of the feature region and the matching length are mutually restricted. In practice, the characteristic region size can be determined according to the limited value of matching length and the maximum drift error of inertial navigation system.

After determining the gravity feature area, the characteristic parameters of the feature area are calculated and judged in order to select the appropriate area as the pre match region.

\section{Characteristics of Gravity Map}

The gravitational field is usually represented by discrete points. The variation law of the gravitational field with the space, including the position information and the intensity information of the gravity field. Assume that the gravity map is a plane grid form, that is, the location point in the gravity map is in the same plane. According to the spatial arrangement of the position points, the grid can be divided into different types.

If the position points are composed of two groups of parallel lines which are perpendicular to each other, they are called regular grids. In the regular grid, the distance between the parallel lines is called a standard grid, otherwise it is not a standard grid. After a certain data processing method, the gravity map can be transformed into the standard grid form [4], and the following discussion is carried out in the standard grid.

The gravity field mainly includes the value of the earth's gravity and local anomaly. The method of using the random field to study the gravity field, and the variation law of the gravity field is described by the statistical parameters Because the spatial variation of the local anomaly field of the earth's gravity is more obvious, it is considered that the local outliers are mainly considered when the statistical parameters are discussed in the characteristic region.
A set of gravity strength $\Omega$ is set to a certain characteristic region $V, V=\{f i, j\}$.

The gravity field intensity $(i, j)$ in the network co-ordinates $f i, j(i, j)$ corresponding to a set of geographical coordinates $(\varphi, \lambda),(i, j) \in \mathrm{U}, \mathrm{U}=\{(i, j) 1 \leq i \leq M, 1 \leq j \leq N\},(M, N)$ is represented by the $\Omega$ size of the characteristic region.

\subsection{Standard Deviation}

Standard deviation $\delta$ is an important index of gravity map, which is defined as:

$$
\begin{aligned}
\delta^{2} & =\frac{1}{M N-1} \sum_{i=1}^{M} \sum_{j=1}^{N}\left(f_{i, j}-\bar{f}\right)^{2}, \\
\bar{f} & =\frac{1}{M N} \sum_{i=1}^{M} \sum_{j=1}^{N} f_{i, j}
\end{aligned}
$$

The standard deviation $\delta$ mainly reflects the intensity of the gravity field and the degree of fluctuation of the whole area. Small standard deviation indicates that gravity map relatively flat, and the fluctuation is not very large, undulating, it will not be conducive for gravity navigation. In general, the ratio of the standard deviation of the magnetic sensor $\delta_{N}$ to the standard deviation $\delta, \delta / \delta_{N}$ is called the signal to noise ratio of the gravity system, the higher the signal to noise ratio, the higher the accuracy of the gravity navigation system.

\subsection{Gravity Entropy}

Entropy is first applied to physical thermodynamics, after a long development has become the basic concept of modern information theory, Navigation is usually carried out in selected gravity information region, the richness of the information or the gravity information can be used to measure the gravitational entropy [2]. Here gravity entropy to measure the gravity field characteristics in the region

Gravitational entropy is defined as

$$
H=-\sum_{i=1}^{M} p_{i} \lg p_{i} \quad p_{i}=\frac{n_{i}}{\sum n_{i}}
$$

Gravitational entropy is defined as follows:

The probability of the emergence of a certain gravity value $p_{\text {i. }}$ In the calculation, the gravity field in the characteristic area is first quantized, so that the number of the gravity field in the range of the gravity field is calculated. It is clear that the magnitude of the gravity entropy is related to the quantization series, the greater the quantization series, the greater the gravitational entropy. In order to make the gravity entropy reflect the characteristics of the gravity field and not be affected by other factors, it should be unified to select the appropriate quantization series. In the practical application, the quantization series should be adapted to the numerical values of the gravity field, and it can be used as a rule of thumb in general. $M=1+3.32 \log N$. In order to prevent the gravity entropy transformation from different feature regions, the above gravity entropy is often used as a unit.

$$
H=-\sum_{i=1}^{M} p_{i} \lg p_{i} / \lg (M)
$$

\section{Volume 5 Issue 2, February 2016}




\section{International Journal of Science and Research (IJSR) \\ ISSN (Online): 2319-7064}

Index Copernicus Value (2013): 6.14 | Impact Factor (2014): 5.611

The gravity entropy reflects the magnitude of the gravity information contained in the characteristic region, and thus reflects the fluctuation characteristics of the gravity field. The greater the gravity entropy, the more uniform the gravity change, the more information the navigation information is. In addition, the gravity entropy also has the ability to smooth measurement error and singular point.

\subsection{Correlation of Distance}

Throughout the Earth's gravitational field near Earth space, its notable feature is its spatial correlation distance close to the two gravity field value is related, but this correlation is relatively limited in a certain range, when the difference between gravity values exceeds a certain distance scales, means the gravity values are not related [5]. Correlation distance, also known as the correlation radius, is the measure of the spatial correlation of the gravity field. When the distance between two points is less than the correlation distance $d$.

There are many methods to calculate the correlation distance, the common methods are: gradient method, the curve fitting method and the maximum likelihood method, the following discussion with the variation function curve fitting method is used to calculate the correlation distance of the gravity field. The theoretical model of variation function is spherical model, exponential model and Gauss model.

$$
r(h)= \begin{cases}C_{0}+C\left[1.5(h / d)-0.5(h / d)^{3}\right] & ; \quad 0 \leq h \leq d \\ C_{0}+C & ; \quad h>d\end{cases}
$$

In the formula, $C_{0}$ called the nugget, $C_{0}+C$ called the base station value for distance $\mathrm{d}$.

Assuming that the gravity field in the feature area $z(x)$ is a stationary random field, the variation function is:

$$
r(h)=\frac{1}{2} E\left\{[Z(x+h)-Z(x)]^{2}\right\}=\frac{1}{2} \operatorname{Var}[Z(x)-Z(x+h)]
$$

So we can get the sample estimate of the variance function of the gravity data in the characteristic region:

$$
\hat{r}(h)=\frac{1}{2 N(h)} \sum[f(x)-f(x+h)]^{2}
$$

When the separation distance is determined, the sample variance function value is generally $h \leq \mathrm{L} / 2$ used, and the $\mathrm{L}$ is the maximum scale of the characteristic region. This is because the measurement data at the large separation distance represent the variance structure of the sample collection edge, rather than the variance structure of the sample stream.

Based on the gravity model and the sample variance function, the estimated value of the correlation radius can be obtained by the regression method. For example, for a spherical model, a variant of the regression function $\mathrm{r}(h)=\mathrm{b}_{0}$ $+\mathrm{b}_{1} h+\mathrm{b}_{2} h_{3}$.

Correlation distance is an important index of the spatial structure of the gravity field. It reflects the cycle of the spatial variation of the gravity field. Large correlation distance, the spatial scale of the change of gravity field is large, the gravity change is more uniform; the correlation distance is small, and the spatial variation of the gravity field is more frequent.

\section{Selection Criteria of Characteristic Region}

In a gravity map, we select several different feature regions. Firstly, we calculate the characteristic of each feature region by the above method, and then use the correlation matching algorithm in this region.

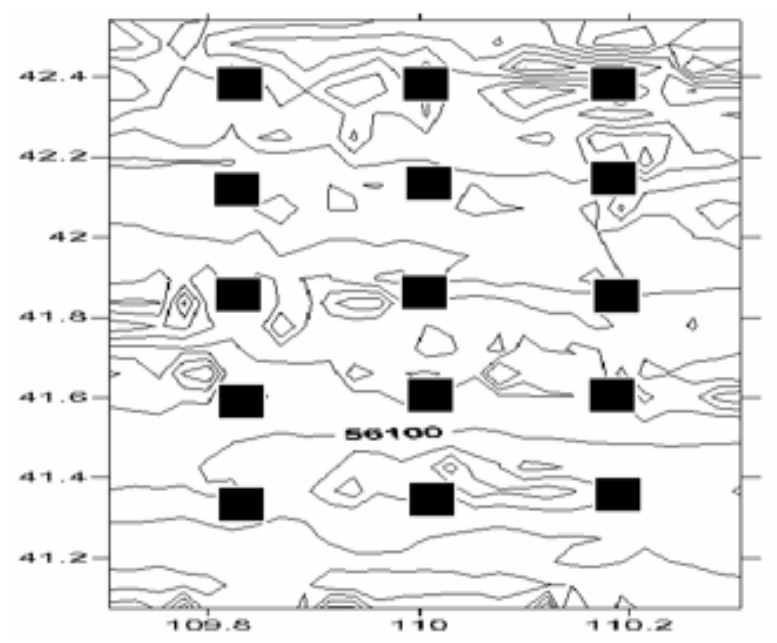

Figure 2: The contour and the gravity field map of the gravity field in a certain area

The gravity map is based on the original gravity field data of a certain area, the data size is $300 * 120$, the grid resolution is about $550 \mathrm{~m} * 550 \mathrm{~m}$, and the contour is shown in Figure 2.15 characteristic regions are selected from the above gravity map, and the grid row and column coordinates of the characteristic regional center are $\{50,100,150,200,250\},\{30$, $60,90\}$, and the characteristic area is 15 grids, as shown in the shadow part of Figure 2. The results presented above is in accordance with the calculation method calculated separately for each feature map parameters are as shown in Table 1, line 4 , in order to discuss the influence of signal to noise ratio (SNR) of matching results, respectively, assumed the three noise of different intensity of noise. Because the gravity map is a standard grid map, the distance is measured by the unit grid distance.

Taking into account the characteristics of gravity in different directions within the feature region is different, along a direction for smoothing the potential limitations, the direction of movement of the carrier and the INS errors are assumed along different orientations. When the simulation is carried out, the carrier is in the same kind of noise level, in the different true trajectory and trajectory of the inertial guidance, a total of 9600 matching calculation is carried out in order to calculate the matching probability and the average matching error, the results are shown in table 1. Matching probability here is the probability that the matching result error is less than the original inertial navigation error, and the original inertial navigation error is set to 4 unit grid distance.

Analysis of the data is in the table, you can see the match parameters of probability and matching error are closely related to the characteristic region.

\section{Volume 5 Issue 2, February 2016}


Table 1: Gravity matching probability and matching error in different feature regions

\begin{tabular}{|c|c|c|c|c|c|c|c|c|c|c|c|c|c|c|c|}
\hline & \multicolumn{3}{|c|}{ Region. 1} & \multicolumn{3}{|c|}{ Region. 2} & \multicolumn{3}{|c|}{ Region. 3} & \multicolumn{3}{|c|}{ Region. 4} & \multicolumn{3}{|c|}{ Region. 5} \\
\hline Gravity Entropy & \multicolumn{3}{|c|}{0.798} & \multicolumn{3}{|c|}{0.755} & \multicolumn{3}{|c|}{0.771} & \multicolumn{3}{|c|}{0.671} & \multicolumn{3}{|c|}{0.867} \\
\hline Correction Distance & \multicolumn{3}{|c|}{15.55} & \multicolumn{3}{|c|}{23.25} & \multicolumn{3}{|c|}{23.66} & \multicolumn{3}{|c|}{16.57} & \multicolumn{3}{|c|}{14.10} \\
\hline Rough Variance Ratio & \multicolumn{3}{|c|}{0.26} & \multicolumn{3}{|c|}{0.29} & \multicolumn{3}{|c|}{0.24} & \multicolumn{3}{|c|}{0.64} & \multicolumn{3}{|c|}{0.19} \\
\hline Signal to No1se Ratio & 7.6 & 5.1 & 3.0 & 10.8 & 7.2 & 4.3 & 10.3 & 6.9 & 4.1 & 7.3 & 4.8 & 2.9 & 5.9 & 3.9 & 2.4 \\
\hline Matching Probability & 0.84 & 0.79 & 0.71 & 0.73 & 0.66 & 0.62 & 0.76 & 0.72 & 0.67 & 0.96 & 0.88 & 0.77 & 0.90 & 0.84 & 0.78 \\
\hline Matching Error & 1.35 & 1.88 & 2.68 & 2.60 & 3.21 & 3.55 & 2.42 & 2.87 & 3.41 & 0.49 & 1.18 & 2.20 & 1.49 & 2.18 & 2.80 \\
\hline
\end{tabular}

\begin{tabular}{|c|c|c|c|c|c|c|c|c|c|c|c|c|c|c|c|}
\hline & \multicolumn{3}{|c|}{ Region. 6} & \multicolumn{3}{|c|}{ Region. 7} & \multicolumn{3}{|c|}{ Region. 8} & \multicolumn{3}{|c|}{ Region. 9} & \multicolumn{3}{|c|}{ Region. 10} \\
\hline Gravity Entropy & \multicolumn{3}{|c|}{0.677} & \multicolumn{3}{|c|}{0.738} & \multicolumn{3}{|c|}{0.570} & \multicolumn{3}{|c|}{0.845} & \multicolumn{3}{|c|}{0.775} \\
\hline Correction Distance & \multicolumn{3}{|c|}{16.49} & \multicolumn{3}{|c|}{13.61} & \multicolumn{3}{|c|}{14.36} & \multicolumn{3}{|c|}{23.70} & \multicolumn{3}{|c|}{15.83} \\
\hline Rough Variance Ratio & \multicolumn{3}{|c|}{0.35} & \multicolumn{3}{|c|}{0.78} & \multicolumn{3}{|c|}{0.67} & \multicolumn{3}{|c|}{0.44} & \multicolumn{3}{|c|}{0.58} \\
\hline Signal to Noise Ratio & 10.1 & 6.7 & 4.0 & 15.8 & 10.5 & 6.3 & 14.1 & 9.4 & 5.6 & 10.4 & 6.9 & 4.1 & 5.4 & 3.6 & 2.2 \\
\hline Matching Probability & 0.76 & 0.68 & 0.0 .57 & 1 & 1 & 1 & 0.99 & 0.95 & 0.86 & 0.94 & 0.85 & 0.74 & 0.99 & 0.93 & 0.86 \\
\hline Matching Error & 2.60 & 3.25 & 4.13 & 0.05 & 0.06 & 0.11 & 0.26 & 0.72 & 1.63 & 0.73 & 1.57 & 2.68 & 0.22 & 0.63 & 1.20 \\
\hline
\end{tabular}

\begin{tabular}{|c|c|c|c|c|c|c|c|c|c|c|c|c|c|c|c|}
\hline & $\operatorname{Re}$ & gion. & 11 & $\operatorname{Reg}$ & gion. & 12 & $\operatorname{Reg}$ & gion. & 13 & $\operatorname{Reg}$ & gion. & 14 & \multicolumn{3}{|c|}{ Region. 15} \\
\hline Gravity Entropy & & 0.853 & & & 0.566 & & & 0.804 & & & 0.821 & & & 0.749 & \\
\hline Correction Distance & & 13.63 & & & 23.08 & & & 27.38 & & & 16.77 & & & 15.33 & \\
\hline Rough Variance Ratio & & 0.63 & & & 0.69 & & & 0.79 & & & 0.76 & & & 0.61 & \\
\hline Signal to Noise Ratio & 4.6 & 3.0 & 1.8 & 6.2 & 4.1 & 2.5 & 8.6 & 5.7 & 3.4 & 11.9 & 7.9 & 4.7 & 17.4 & 11.6 & 7.0 \\
\hline Matching Probability & 0.97 & 0.98 & 0.93 & 0.94 & 0.86 & 0.75 & 1 & 0.97 & 0.85 & 1 & 0.99 & 0.95 & 1 & 0.99 & 0.98 \\
\hline Matching Error & 0.32 & 0.43 & 0.90 & 1.03 & 1.79 & 2.73 & 0.12 & 0.44 & 1.49 & 0.10 & 0.20 & 0.59 & 0.10 & 0.18 & 0.38 \\
\hline
\end{tabular}

The greatest influence on the matching probability and matching error is the ratio of signal to noise ratio and the ratio of rough to noise ratio, the greater the SNR, the larger the ratio of rough to noise ratio, the higher the matching precision. Therefore, when selecting the characteristic region of the match, the gravity field should be chosen to be larger, and the region of local variation also large.

Secondly, the gravity entropy and the correlation distance have effect on the match result, the greater the gravity entropy and the correlation distance, the better the reliability of the matching results. Feature selection criteria: after a lot of simulation test and theoretical analysis, can be summed up the following characteristics of the selection criteria

$$
\delta / \delta_{N}>5, \sigma / \delta>0.6, H>0.7, d / L>2
$$

In the above simulation experiment, the selection criterion is satisfied. Within the region, the matching probability will be greater than $95 \%$, matching The mean error is controlled within 0.3 cell grids, Can meet the general engineering application requirements.

\section{Conclusion}

The selection of the characteristic area of the gravity map is an important aspect of gravity navigation technology. To obtain excellent performance in gravity navigation, features selection must be carried out in advance in the region. This paper is mainly from the statistical point of view, different statistical parameters are used to describe the characteristics of the gravity map of all aspects, including the degree of gravity value dispersion, the degree of smoothness, the correlation characteristic and the information richness degree and so on. Start with these parameters, According to the experiments carried out on 15 gravity maps of different characteristics, the selection criterion function of gravity map is constructed.

Under this criterion, the performance of the gravity navigation system is obtained, which meets the general requirements of navigation. This criterion can be used as a reference for the selection of gravity navigation feature area.

\section{References}

[1] Ingemar Nygren, Magnus Jannon. Terrain Navigation Using the Correlator Method [J]. Royal Institute of Technology, 2004: 649-657

[2] A Popoulis. US. Pillai. Probability Random Variable and Stochastic Process [M]. Polytechnic University, 2004, 5111-51

[3] James C.H Chu, Wen Chien His, Lincoln Hubbard. Performance of magnetic field-guided navigation system for interventional neurosurgical and cardiac procedures [J]. Journal of Applied Clinical Medical Physics, Volume 6, Number 3, Summer 2005

[4] Saemus Timothy Tuoty. Geophysical map representation, Abstraction and interrogation for autonomous underwater Vehicle [D]. A dissertation of the PH.D at the Massachusetts Institute of Technology, 1994: 1-158. 


\section{International Journal of Science and Research (IJSR) \\ ISSN (Online): 2319-7064}

Index Copernicus Value (2013): 6.14 | Impact Factor (2014): 5.611

[5] Liu Chun-yuan,Yan Shu-wang. Relative Distance Discrimination on the Random Field. Journal of Tianjin University2003, Vol.36 No.1, 58-62

[6] Ratkovic J A. Estimation techniques and other work on Image correlation [A]. ICSP Processings. 1997, 2:54-60.

[7] Liu Ying Zhang Ji-xian, Liu Jian. Terrain information Analysis on Terrain Navigation Remote Sensing Information, 2000, 2:21-23

[8] Barid C A, Abramson M R. A comparison of several digital map-aided navigation techniques [J]. New York: The Institute and Electronics Engineers Inc, 1984, 231238

[9] Wu Cheng-fu Ma Song-hui, Chen Huai-min. A Method for confidence Analysis of Terrain Match Journal of Northwestern Polytechnic University, 2004, v ol.22 No.3, 346-349 